\title{
Dens invaginatus: A report of two cases
}

\section{(1) Mohamed Faizal Asan, (1) G. Subhas Babu, (1) Supriya Bhat, (1) Ananya Madiyal, (1) Vaibhav Pandita}

Nitte (Deemed to be University), AB Shetty Memorial Institute of Dental Sciences (ABSMIDS), Department of Oral Medicine and Radiology, Mangalore, India

\section{Date submitted:}

19.04.2020

Date accepted:

28.05.2020

Online publication date:

15.03.2021

\section{Corresponding Author:}

G. Subhas Babu Professor, Nitte (Deemed to be University), AB Shetty Memorial Institute of Dental Sciences (ABSMIDS), Department of Oral Medicine and Radiology, Mangalore, India

goginenisb@yahoo.co.in

ORCID:

orcid.org/0000-0001-9383-7886

Keywords: Dens in dente, classification, tooth abnormalities, incisor, dental papilla

\begin{abstract}
Dens invaginatus is an uncommon developmental anomaly of teeth arising due to the invagination of the dental papilla. The affected teeth show an infolding of enamel and dentine even sometimes extending into the root causing perforation. The malformation exhibits a wide variety of presentation. On the other hand, it can affect any teeth including primary, permanent and supernumerary teeth. The frequently affected teeth are maxillary lateral incisors. It usually results in pulpal necrosis when the invagination is extending into the pulp chamber. In most cases, it is diagnosed as an incidental finding in a routine dental examination as an alteration of the crown structure or as a radiographic finding. An early prophylactic approach is the most effective means of treatment. However, endodontic management of the affected tooth is often required.
\end{abstract}

\section{Introduction}

"Dens invaginatus" is a form of tooth dysmorphogenesis formed mainly due to the infolding of enamel and dentin or inflection of the lingual pit of an incisor before calcification. This form of dental anomaly which is also termed as dens in dente or dilated composite odontome was first reported in human tooth by Ploquet in 1794 (1). Different causes have been attributed to this anomaly; Kronfeld in 1934 put forth the theory of focal growth retardation, Fischer and Sprawson attributed infection as a cause in 1936 and 1937, respectively. In 1937, Rushton attributed it to a rapid proliferation of a part of the inner enamel epithelium into the developing dental papilla, and Euler in 1939 and Atkinson in 1943 stated an increased localized external pressure as a cause. According to Bruszt in 1950, it may be a result of two tooth germs fusing together. Gustafson and Sundberg stated traumatic injury as a probable etiology. In 1957, according to Oehlers, the distortion of enamel organ might result in malformation. The absence of signaling molecules for morphogenesis and genetic factors was also reported as a causative factor $(2,3)$. Though it can affect primary, permanent and supernumerary teeth, the most frequently affected teeth are the lateral incisors in the maxillary arch. This may be due to the effect of external force exerted on the lateral incisor tooth bud by the adjacent teeth during the development (4).

The prevalence rate of dens invaginatus is about $0.04 \%$ to $10 \%$. Male predilection with a ratio of $3: 1$ is noted (5). There are numerous classifications that were put forward to describe the different forms of dental invaginations, but Oehler's classification, which is based on radiological criteria, is widely used. Oehler has classified the anomaly into three variants depending on the extent of invagination (6).

Type 1: Minor form of coronal enamel lining that does not extend beneath the cemento-enamel junction (CEJ). 
Type 2: Form of enamel lining extending into the root but limited to a blind sac, that may or may not be communicating with the dental pulp.

Type 3 A: Invagination having no communication with the pulp but extends into the root forming a lateral communication with the periodontal ligament space by a pseudoforamen.

Type 3 B: Invagination completely lined by enamel and sometimes cementum, extending into the root, perforating at the apex by a pseudoforamen.

\section{Case Presentations}

\section{Case 1}

A 20-year-old female was admitted to our department with a complaint of pain in her right upper anterior tooth for a month. The pain was pricking type, intermittent in nature, aggravated during mastication and relieves on its own and non-radiating in nature. On intraoral examination, the right maxillary lateral incisor appeared to have an altered morphology (Figure 1A). There was no carious lesion present on the tooth surface, but the tooth showed tenderness on vertical percussion. Based on the findings of the clinical examination, it was pre-diagnosed as apical periodontitis. Informed consent was obtained before taking clinical photographs of the patient.

Intraoral periapical radiographic (IOPAR) examination of the right maxillary lateral incisor revealed altered morphology with a coronal enamel invagination that was parallel to the pulp canal

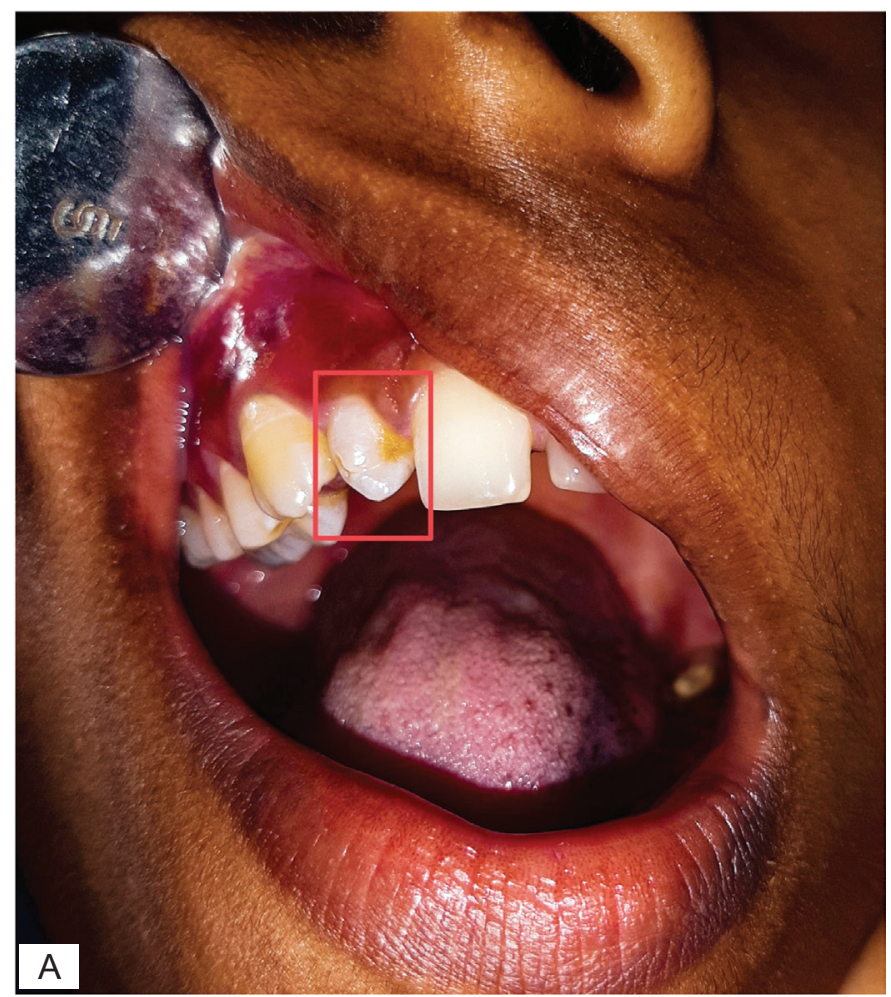

and perforated the root laterally (Figure 1B). The periapical area showed an ill-defined radiolucency similar to rarefying osteitis. Based on the radiographic appearance, a confirmed diagnosis of dens invaginatus (type 3 A) was established. Endodontic therapy of the tooth was planned but the patient did not turn up for further treatment.

\section{Case 2}

A 19-year-old male was admitted to our department with a complaint of abnormal shape of the left upper anterior tooth since its eruption, with no history of pain and symptoms. On intraoral examination, crowding of the maxillary central incisors was noted and the left maxillary lateral incisor displayed a different morphology with a pit in the incisal edge of the tooth. There was no evident carious lesion on the tooth surface (Figure 2A). Informed consent was obtained before taking clinical photographs of the patient.

IOPAR of the left maxillary lateral incisors revealed a coronal enamel invagination that extended to the CEJ (Figure 2B). Based on the radiographic feature, a definite diagnosis of dens invaginatus (type 1) was established. Prophylactic sealing of the invagination of the tooth was planned.

\section{Discussion}

Dens invaginatus is diagnosed as an incidental finding in a routine dental examination as a difference in the crown structure or as a radiographic finding. The altered crown morphology or a

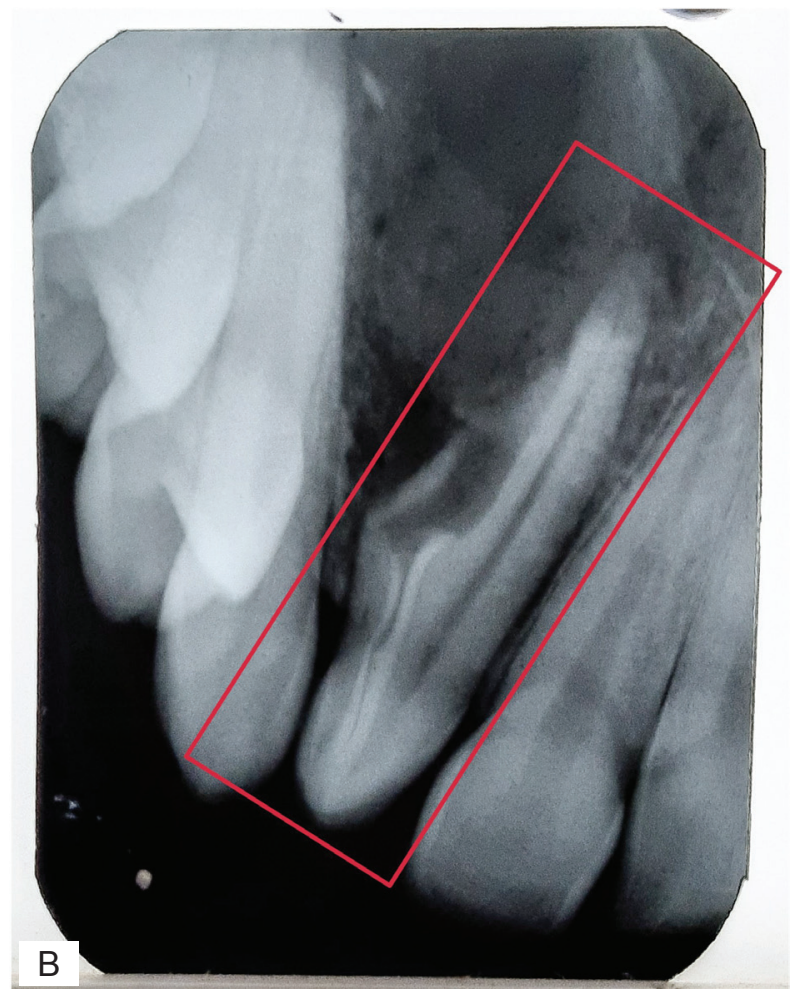

Figure 1. A) Right maxillary lateral incisor with an altered morphology of the clinical crown. B) Intraoral periapical radiographic of right maxillary lateral incisor showing a deep invagination with lateral perforation on the distal aspect of the root and a small periapical radiolucency 

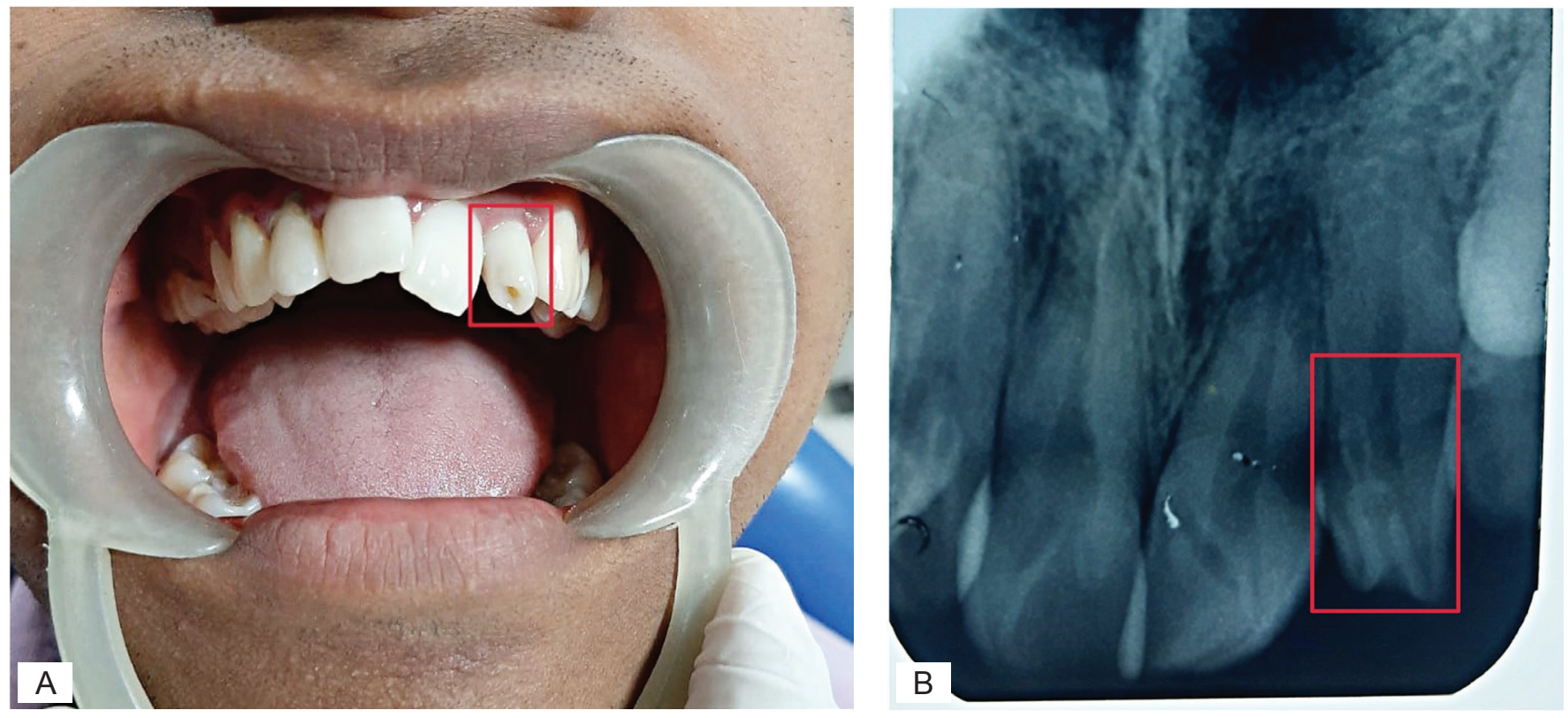

Figure 2. A) Altered morphology of the clinical crown of left maxillary lateral incisor with a deep pitting on the incisal aspect. B) Intraoral periapical radiographic showing coronal enamel invagination in the maxillary left lateral incisor extending downwards till the cemento-enamel junction

deepened foramen coecum may help in the clinical identification, but sometimes the affected teeth may not show any clinical alteration of the crown. In most cases the affected crown may be in conical or barrel-shaped. The altered or conical shape of the incisors may lead to significant esthetic discomfort (7). Early diagnosis is important to determine the necessary treatment because coronal invaginations may extend into the pulp or may allow the entry of irritants into the pulp or periapical area. In most cases, the pulp is separated from the invagination by a thin layer of enamel and dentine, so the invasion of the bacteria or irritants through the invagination may reach the pulp easily and may predispose to pulpal and periapical pathology (8).

Here in the first case, there was altered crown morphology with perforation of root structure, whereas the second case had coronal malformation without any symptoms. Undiagnosed and untreated coronal invaginations may lead to periapical pathology as noticed in our case. The management of dens invaginatus involves prophylactic closure or sealing of the invagination, non-surgical endodontic therapy, endodontic surgery (9), extraction with intentional replantation (10), based on the type of invagination.

\section{Conclusion}

A thorough knowledge of the clinical views and radiographic presentation of dens invaginatus may help for the identification of the case. There are various classifications available in the literature, describing the different forms of invaginations. They may help us to understand the various presentations of dens invaginatus, enabling early diagnosis and providing appropriate treatment measures.

\section{Ethics}

Informed Consent: Informed consent was obtained before taking clinical photographs of the patient.

Peer-review: Externally peer-reviewed.

\section{Authorship Contributions}

Surgical and Medical Practices: M.F.A., G.S.B., Concept: M.F.A., Design: S.B., Data Collection or Processing: A.M., Analysis or Interpretation: G.S.B., Literature Search: A.M., V.P., Writing: M.F.A., S.B.

Conflict of Interest: No conflict of interest was declared by the authors.

Financial Disclosure: The authors declared that this study received no financial support.

\section{References}

1. Schulze C. Developmental abnormalities of the teeth and jaws. Thoma's oral pathology. 6th ed. St Louis, MO: Mosby Company; 1970:96-183.

2. Rushton MA. A collection of dilated composite odontomas. Br Dent J. 1937;63:65-85.

3. Casamassimo PS, Nowak AJ, Ettinger RL, Schlenker DJ. An unusual triad: Microdontia, taurodontia, and dens invaginatus. Oral Surg Oral Med Oral Pathol. 1978;45:107112.

4. Munir B, Tirmazi SM, Majeed HA, Khan AM, Iqbalbangash $N$. Dens invaginatus: Aetiology, classification, prevalence, diagnosis and treatment considerations. Pakistan Oral Dent J. 2011;31:191-198.

5. Bishop K, Alani A. Dens invaginatus. Part 2: clinical, radiographic features and management options. Int Endod J. 2008;41:1137-1154. 
6. Oehlers FA. Dens invaginatus (dilated composite odontome). I. Variations of the invagination process and associated anterior crown forms. Oral Surg Oral Med Oral Pathol. 1957;10:1204-1218.

7. Aydın N, Karaoğlanoğlu S, Özcivelek Mersin T, Aybala Oktay E, Demir F. Aesthetic restoration of peg lateral incisors with direct composite resin. Gulhane Med J. 2019;61:25-27.
8. Hülsmann M. Dens invaginatus:aAetiology, classification, prevalence, diagnosis, and treatment considerations. Int Endod J. 1997;30:79-90.

9. Halawar SS, Satyakiran G, Krishnanand P, Prashanth R. Dens invaginatus (dilated odontome) in mandibular canine. J Oral Maxillofac Pathol. 2014;18(Suppl 1):157-162.

10. Girsch WJ, McClammy TV. Microscopic removal of dens invaginatus. J Endod. 2002;28:336-339. 